\title{
Surveillance for Mass Gatherings: NCAA Final Four 2017 in Maricopa County, Arizona
}

\author{
William E. Smith*1, Kate Goodin', Rasneet S. Kumar', Vjollca Berisha1, Craig Levy¹, \\ Siru Prasai ${ }^{1}$ and Kristen Pogreba-Brown²
}

${ }^{1}$ Office of Epidemiology, Maricopa County Department of Public Health, Phoenix, AZ, USA; ${ }^{2}$ University of Arizona, School of Public Health, Tucson, AZ, USA

\section{Objective}

To describe and present results for the enhanced epidemiologic surveillance system established during the 2017 National Collegiate Athletic Association Division I Men's College Basketball Championship (Final Four) events.

\section{Introduction}

Final Four-associated events culminated in four days of intense activity from March 31st through April 3rd, and added an estimated 400,000 visitors to Maricopa County's 4.2 million residents.

\section{Methods}

Preparation included: refinements in enhanced surveillance for previous events (including Super Bowl XLII); a rehearsal on information sharing for team leads; just in time training for field team members; a tabletop exercise on $2 / 22$; and solicitation of lessons learned from jurisdictions recently hosting the Final Four.

Enhanced surveillance began on $3 / 24$ and continued through $4 / 10$ (one week before the first major event until one week after the championship game) with intensified surveillance from 3/31-4/3. Subject matter experts for each enhanced surveillance component functioned as team leads. A surveillance coordinator was assigned to review data and prepare reports. Team members were sent a plan of the day detailing daily surveillance activities.

An enhanced surveillance (SURV) alert requesting an increased index of suspicion for events of public health significance was sent to pre-established lists of healthcare providers. Urgent care clinics within five miles of venues were asked to report influenza-like, gastrointestinal, rash, and neurological illness visits daily. Emergency department records in the National Syndromic Surveillance Program, Electronic Surveillance System for Early Notification of CommunityBases Epidemics (ESSENCE) were monitored daily for influenza-like illness, gastrointestinal illness, injury, records of interest, heat-related illness and event-specific terms. Mumps and meningitis were added after outbreak reports were received from home jurisdictions of Final Four teams. Death certificate data, Office of the Medical Examiner line lists and preliminary reports of death were reviewed daily for reportable diseases or circumstances of public health significance. Communicable disease data was reviewed daily for notifiable disease cases of concern, aberration detection as compared to the previous four years, outbreak review, and Influenza-like-illness.

Field teams of staff and volunteers were deployed to three days of Music Fest, four days of Fan Fest, and three Final Four games. Attendees presenting to first aid stations were requested to complete an electronic questionnaire capturing illness and injury syndromes. These were submitted and epidemiologically assessed in near-realtime. Syndrome-specific data were geo-located on venue maps during events to identify spatial clustering. Patient Presentation Rates (PPR) and Transport to Hospital Rates (TTHR) per 10,000 attendees were calculated.

To enhance animal health system surveillance, veterinarians and agencies that work with animals were notified to increase the index of suspicion for unusual animal disease, keep alert for outbreaks with zoonotic potential, and update 24/7 emergency contact lists.
Health-related media reports, Final-Four-specific reports, healthaggregated Twitter reports, and breaking news alert subscriptions were monitored. Poison Control Center (PCC) reports were assessed by conducting regular queries of the National Poison Data System (NPDS). Reports from the 24/7 Disease Reporting Line were monitored.

A one page enhanced surveillance report was developed for daily distribution to inter-disciplinary partners; a more detailed report was distributed to health and medical partners. Physicians overseeing the health/medical care of teams were included in information sharing. Public health intelligence information was exchanged with epidemiologists from home jurisdictions of Final Four teams.

\section{Results}

301 field questionnaires were completed, including 146 from Final Four games, 127 from the Music Fest, and 28 from the Fan Fest. Final Four games experienced a PPR of 9.5, and a TTHR of 0.52. Music Fest results were a PPR of 9.4, and a TTHR of 0.15. For the Fan Fest, there was a PPR of 5.5, and a TTHR of 0 .

PCC data review resulted in investigation of four cases for potential ricin exposure. These reports were determined to be exposure to castor beans and the castor bean plant (Ricinus communis) only. One report indicating potential phosgene occupational exposure to an air conditioning system worker was reviewed, and judged unlikely to cause noted symptoms.

Outbreak information from home jurisdictions of Final Four teams resulted in increased index of suspicion for mumps, additional surveillance and mentions in media surveillance reports.

Review of communicable disease, mortality, and ESSENSE data resulted only in routine investigations.

\section{Conclusions}

Surveillance information from disparate surveillance systems was synthesized into reports which enhanced health and medical situational awareness and information sharing; interdisciplinary partners highlighted the utility of the one-page report. Enhanced surveillance allowed the rapid identification and characterization of potential threats, and provided an evidence base for public health decisions. Establishment of field teams allowed for near-real-time tracking of patient presentations and transports and rapid identification and characterization of syndromes of concern and potential threats. Public health intelligence information exchange with home jurisdictions of Final Four teams resulted in targeted surveillance for mumps and meningitis.

\section{Keywords}

mass gathering; syndromic surveillance; public health surveillance; epidemiological surveillance; Information sharing

\section{Acknowledgments}

Thank you to the 2017 NCAA Final Four enhanced surveillance team.

\section{*William E. Smith}

E-mail: williamsmith@mail.maricopa.gov 